\title{
Significance test for seismicity rate changes before the 1987 Chiba-toho-oki earthquake ( $M$ 6.7) Japan
}

\author{
Kenji Maeda and Stefan Wiemer \\ Seismology and Volcanology Research Department, Meteorological Research Institute, \\ Tsukuba, Japan
}

\begin{abstract}
A precursory seismic quiescence lasting $1.5 \pm 0.5$ years was observed prior to the $1987 M 6.7$ Chiba-toho-oki earthquake, Central Japan. This event was the largest mainshock to occur in the region in 60 years. A quantitative analysis of the seismicity rates, using two independent catalogs provided by the NIED and JMA networks, shows that the precursory seismic quiescence is centered in the shallower part of the rupture zone of the subsequent mainshock, at a depth of 20-40 km. At the hypocenter of the 1987 Chiba-toho-oki mainshock, a 50\% increase in the seismicity rate was detected in the NIED data, coinciding in time with the onset of quiescence $(1986.4 \pm 0.5)$. The simultaneous appearance of both quiescence in the shallow part of the rupture zone observed in two catalogs, and a rate increase in the immediate hypocenter region, suggest that these phenomena are causally linked to the subsequent mainshock. However, a quantitative analysis of both catalogs reveals that the precursory quiescence and rate increase are not unique, since rate changes of this duration and significance often occur in the data. A rate change of this significance rating could probably not be detected as a precursor in a real time approach. For the aid of real time monitoring of seismicity rate changes, we introduce the method to calculate the 95-percentile of confidence level for the significant rate changes.
\end{abstract}

Key words quiescence - seismicity rate increase quantitative analysis - significance test - the 1987 Chiba-toho-oki earthquake

\section{Introduction}

A change in stress within or near a seismogenic volume in the Earth's crust produces a change in the rate of micro-earthquakes. Aftershocks are the most obvious phenomena of this kind: a dislocation step-function (i.e. an earth-

Mailing address: Prof. Kenji Maeda, Seismology and Volcanology Research Department, Meteorological Research Institute, 1-1 Nagamine, Tsukuba, 305-0052, Japan; e-mail: kmaeda@mri-jma.go.jp, stefan@seismo.ifg.ethz.ch quake) produces numerous aftershocks, but also areas of decreased seismicity rate (e.g., Stein et al., 1992; Dieterich, 1994; Toda et al., 1998). Triggered seismicity foreshocks and quiescence are other examples of seismicity rate changes caused by stress changes. Therefore, seismicity rates can act as an indirect remote stress sensor at depth, where no direct measurements exist. Possible precursory seismicity rate or stress changes are of great interest for the understanding of rupture mechanics, but are not easily identified mainly because of the 'noise' in seismicity catalogs due to frequent man-made changes in rate. A rigorous statistical analysis of possible precursory rate changes is needed to establish that these signals are indeed causally linked with an upcoming mainshock and not a coinci- 
dental correlation of random or systematic noise in the data.

There are numerous reports related to both qualitatively and quantitatively analyzed precursory seismic quiescence (e.g., Inouye, 1965; Mogi, 1969, 1985; Yamashina and Inoue, 1979; Noguchi, 1983; Wyss, 1986; Wyss and Burford, 1987; Kisslinger, 1988; Reasenberg and Matthews, 1988; Wyss and Habermann, 1988; Ogata, 1992; Wiemer and Wyss, 1994; Dieterich and Okubo, 1996; Wyss et al., 1996, 1997, 1999; Yoshida et al., 1996; Wyss and Wiemer, 1997). However, to estimate the usefulness of precursory seismic quiescence for predicting large earthquakes, more case studies for different tectonic regimes that apply an objective and quantitative analysis are needed. Unfortunately, inhomogeneities in earthquake catalogs, which are inevitably introduced by changes in the observational and analyzing system, frequently produce artificial rate changes (e.g., Habermann, 1987; Wyss, 1991; Eneva et al., 1995; Zuniga and Wyss, 1995). Therefore, a careful evaluation of the homogeneity of the catalog under investigation is needed to obtain reliable results. In this study, we used two independent catalogs, which cover the Kanto area in Central Japan. The catalogs are produced by: 1) the Japan Meteorological Agency (JMA) and 2) the National Research Institute for Earth Science and Disaster Prevention (NIED). Objective and quantitative analysis of quiescence requires clearly defined standards for measuring the rate change of seismicity and we examined the data with an 'ZMAP' computer program (Wiemer and Wyss, 1994; Wyss and Wiemer, 1997) which provides an effective tool to make such an analysis.

According to the JMA catalog, the 1987 Chiba-toho-oki earthquake ( $M$ 6.7) occurred on December 17 at a depth of $58 \mathrm{~km}$ off the east coast of Chiba Prefecture in the Kanto district, Central Japan (fig. 1). This earthquake represents the largest mainshock in this area for the last 60 years, and is thought to have occurred as a consequence of the internal deformation of the Philippine Sea plate. The fault plane strikes in NNW-SSE direction, dips steeply eastward and displays almost right-lateral faulting (Okada and Kasahara, 1990). Odaka and Maeda (1994) analyzed the University and JMA catalogs and reported that about one year prior to the occurrence of this mainshock, the seismicity rate decreased at shallow depths, and increased in the immediate hypocentral area. However, no quantitative analysis was performed in their study and thus it is not clear if the change in rate of earthquakes is significant compared to the background seismicity fluctuations in this area.

The main objectives of our study are to reevaluate the rate changes before the 1987 Chibatoho-oki earthquake described by Odaka and Maeda (1994), using both the JMA and NIED catalogs. This includes a quantitative evaluation of the significance of the precursory rate changes. In addition, we present results from the analysis of catalog homogeneity. Finally, we discuss the implications of our results for future monitoring of seismicity rate changes in the Kanto and other areas.

\section{Data}

The area investigated is part of the Kanto district, Central Japan (fig. 1). Seismic monitoring in this densely populated and heavily industrialized region is extensive, and we are in the fortunate situation of having access to the data from two seismic networks. We use the two independently determined hypocenter catalogs of JMA and NIED, limited to earthquakes with depth $\leq 100 \mathrm{~km}$ and the period 1979.5-1997.0. The JMA catalog includes moderate and large earthquakes that occurred in and around Japan, and is widely used for analyzing seismicity in Japan. However, the quality and completeness level changes considerably as a function of time and space (Ishikawa, 1987). The NIED catalog includes smaller events than the JMA catalog in the Kanto area, because of the greater density of seismometers of the NIED network (Okada, 1984). Equally important, our preliminary quality comparison shows that the reporting as a function of magnitude, time, and space is more consistent, and hypocenter locations are determined more accurately in the NIED data than in the JMA data. Our study of the significance of the precursory rate changes before the 1987 Chiba-toho-oki earthquake is thus mainly based on the NIED data. 




Fig. 1. Map of the Kanto region, Central Japan. Epicenters are shown as black dots. The thick line shows the location of the EW and NS trending, $35 \mathrm{~km}$ wide, cross-sections analyzed in figs. 3 and 4 . A star marks the epicenter of the 1987 Chiba-toho-oki earthquake ( $M 6.7)$, and aftershocks within a 30 -day period are marked as gray circles.

We exclude events marked as quarry blasts in the NIED catalog from the analysis. However, in some regions many blasts remain in the data. We test for the presence of quarry blast by plotting histograms of the distribution of events as a function of the hour of the day. Regions with quarry blasts show an increase in activity around 12:00 and 18:00 h. Regions not containing blasts, on the other hand, do not display these peaks, but show the generally observed decrease in the number of detected events during daytime hours due to the higher seismic background noise. Blasts are concentrated ai shallow depths, but can be detected as deep as $40 \mathrm{~km}$ in some regions. Wyss and Wiemer (1997) decided to exclusively analyze nighttime events in their analysis of the shallow seismicity in the Kanto region. However, to maximize the amount of data available, we use all hours but exclude the regions containing blasts.

We investigate the lower threshold of the magnitude of completeness $\left(M_{c}\right)$ as a function of time for the entire study area outlined in fig. 1. $M_{r}$ is approximated by computing the point of maximum curvature of the cumulative frequency-magnitude distribution. Results of this anal- 

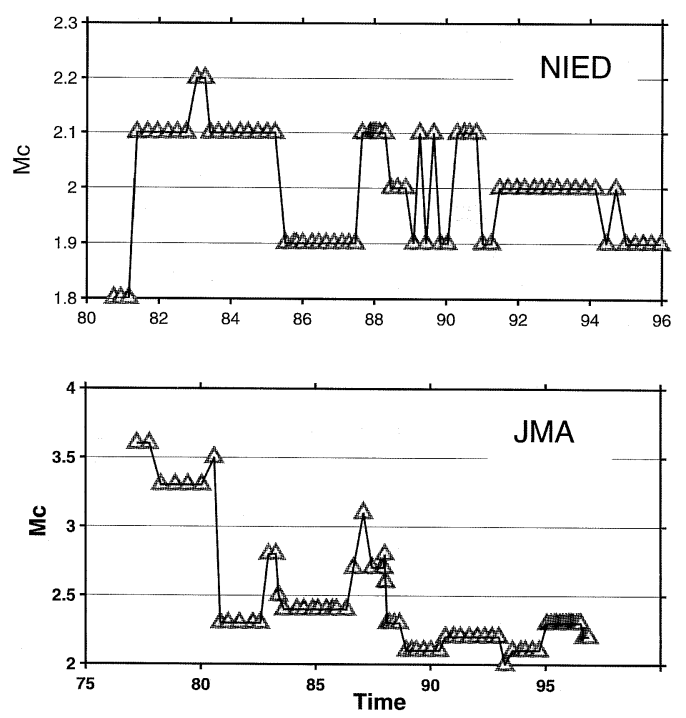

Fig. 2. Magnitude of completeness $\left(M_{c}\right)$ as a function of time for the NIED (top) and JMA (bottom) data.

ysis are shown in fig. 2. From these figures, we determine the minimum usable $M_{c}$ for a homogeneous reporting to be $M_{c}=3.0$ for the JMA catalog for the period after 1981 , and $M_{c}=2.2$ for the NIED data set after 1979.5. Note that $M_{c}$ for the NIED data set remains approximately constant with time, whereas a drop from $M_{c}=3$ to $M_{c}=2.2$ is observed in the JMA data, a result of frequent network improvements.

To separate the dependent from the independent seismicity, aftershock sequences can either be modeled using the modified Omori Law or an Epidemic Type Aftershock Sequence (ETAS) approach (e.g., Ogata, 1992) or removed by declustering the catalog. The fundamental philosophy of the two approaches is the same, but the techniques differ. We chose to decluster the catalog, using Reasenberg's algorithm (Reasenberg, 1985). The parameter values used in the declustering algorithm are the original ones derived by Reasenberg for California (Reasenberg, 1985). After declustering, we notice that the aftershocks of the 1987 Chibatoho-oki event are not entirely removed. This is commonly observed for large mainshocks with an extended rupture area. The increased seismicity rate during the aftershock period influences the calculation of the $Z$-value because it changes the mean rate and the variance of the background seismicity rate. Rather than applying a more rigid declustering, we decided to eliminate the entire aftershock period by eliminating the 4.04 years after the mainshock from our analysis (1987.96-1992.0). We also confirmed our results by analyzing pre-mainshock seismicity and obtained generally comparable results. The results obtained in this paper are mostly based on the declustered and time-cut catalogs, however, we confirm that our results are independent of the declustering by also evaluating the undeclustered, original catalog.

\section{Method}

The hypothesis we consider is the one formulated by Wyss and Habermann (1988). The precursory seismic quiescence hypothesis assumes that some mainshocks are preceded by seismic quiescence, which is a decrease of the mean seismicity rate, as compared to the preceding, declustered background rate in the same crustal volume. The rate decrease, which must be judged significant by some clearly defined standard, takes place within part, or all, of the source volume of the subsequent mainshock. It extends up to the time of the mainshock, or may be separated from it by a period of foreshock activity, and the rate decrease takes place in all magnitude bands. We also studied the hypothesis that a significant rate increase in the hypocentral region preceded the 1987 mainshock. To test this hypothesis, we applied analysis techniques analogous to those used to investigate rate decreases.

To establish whether the 1987 Chiba-tohooki earthquake was preceded by a significant or even unique precursory seismic rate change we performed the following analysis steps:

- Compare the background seismicity rate with the rate in the period before the mainshock. By using Z-value cross-sections through the 1987 hypocenter, we optimized the rate change and thus define its characteristics (significance, duration and spatial extent). 
- Compute the likelihood of random occurrence of a rate change with the optimized characteristics. This addresses the question of how likely a rate change with a certain significance is to be created by chance in the data set.

- Using the alarm cube approach, we evaluated how frequently a rate change with the optimized characteristics, at least as significant as the precursory one, occurs in the selected crosssections. A significant quiescence would pass all of the above steps. A unique quiescence ranks in addition as the highest-ranking quiescence in the data. The techniques involved in each of these steps have been described in length in a number of publications (Wiemer and Wyss, 1994; Wiemer, 1996; Wyss and Wiemer, 1997, Wyss et al., 1999) and we will only briefly summarize them here. A fourth step would be required to simulate that a rate change could be detected in real time monitoring. Since the characteristics and location of a future rate change are unknown, the optimized parameters cannot be used and consequently one needs to perform a grid search over the entire parameter space to identify all alarms above a given significance in the six-dimensional parameter space ( 3 in space, time, $N, T_{w}$ ).

We analyzed a $35 \mathrm{~km}$ wide, NS and EW trending cross-section through the 1987 mainshock hypocenter. Changes in the seismicity rate were evaluated as a function of time at every node of a densely spaced two-dimensional grid (distance, depth) with $3 \times 3 \mathrm{~km}$ spacing. At each node, the data analyzed were the nearest $N$ events (e.g., $N=100$ ), and a window (e.g., $T_{w}=1.5$ years) was moved through the time series, stepping forward by one sampling interval (one month). For each window position, we calculated the standard deviation $Z$, generating the function LTA $(t)$ (Wiemer and Wyss, 1994), which measures the significance of the difference between the mean seismicity rate within the window, $R_{2}$, and the background rate, $R_{1}$, here defined as the mean rate outside the window, but in the same volume. The $Z$-value was defined as

$$
Z=\left(R_{1}-R_{2}\right) /\left(S_{1} / n_{1}+S_{2} / n_{2}\right)^{1 / 2}
$$

where $S_{1}, S_{2}$ and $n_{1}, n_{2}$ are the variances and numbers of samples, for outside and within the window, respectively. The resulting three-dimensional (distance, depth, time) matrix of $Z$-values constituted the basis of our quantitative analysis of rate changes.

The radii of the crustal volumes sampled were variable and inversely related to the local seismicity rate because we kept $N$ constant to satisfy assumptions for statistical evaluations. The Z-maps show the local relative strength of rate changes at the time of the window position selected. By definition (eq. (3.1)) a positive $Z$-value indicates a decrease in the seismicity rate, and is mapped in red. In animated time slices, we viewed $Z$-maps for all possible window positions in time, and a summary of all values of $Z$ above a selected alarm level was given in the alarm-cube representations of the results (Wiemer, 1996; Wyss and Wiemer, 1997; Wyss et al., 1999). Thus, we identified all alarms above a given significance threshold that may be present in a data set.

In order to judge the significance of $Z$-values calculated for a particular data set, we generated synthetic $Z$-value matrices based on a random selection of samples from the earthquake cata$\log$ of the cross-section area studied. $N$ events were extracted from the catalog at random, regardless of location, but retaining the origin time, and a LTA $(t)$ function for a given window length (e.g., $T_{w}=1.5$ years) was calculated. Repeating this process 1200 times resulted in a matrix equal in size to the one obtained with the grid size used in this paper. Such synthetic matrices were calculated 1000 times and the maximum $Z$-value, $Z_{\max }$, was retained each time. The significance of a $Z$-value obtained in the analysis of the real data was judged in comparison to this synthetic $Z_{\max }$ distribution (Wiemer, 1996; Wyss et al., 1996).

\section{Results}

\subsection{Seismicity rate changes before the 1987 Chiba-toho-oki earthquake}

We computed NS and EW trending Z-value cross-sections through the hypocenter of the 
mainshock (marked in fig. 1), with $35-\mathrm{km}$ width for both the NIED $(M \geq 2.2)$ and JMA $(M \geq 2.5)$ catalogs. For the JMA data we used a magnitude cut-off lower than $M_{c}$ because magnitude shifts occur in the data, as discussed below, and cutting at $M$ introduces artifacts in the seismicity rates. The width of $35 \mathrm{~km}$ was chosen to contain the aftershocks of the 1987 mainshock. Hypocenters are plotted in figs. 3 and 4 for the NIED and JMA data respectively. The aftershock locations within a 30 -day period are marked in red. The rupture area outlined by the aftershock activity is located at a depth of 20-50 $\mathrm{km}$ and the hypocenter is located at a depth of $47 \mathrm{~km}$ (NIED data) and $58 \mathrm{~km}$ (JMA data) respectively. The NIED data set contains a total of 3912 events in the NS, and 2527 events in the EW cross-section. Based on these cross-sections (top row figures in figs. 3 and 4), we compared the seismicity rate in the 1.5 years prior to the 1987 Chiba-toho-oki earthquake
(1986.46-1987.96) with the background seismicity rate (1979.5-1997.0, but excluding the periods 1986.46-1987.96 and 1987.96-1992.0). At each grid-node, the 100 closest earthquakes were sampled. The average radius of the volumes sampled was $12 \mathrm{~km}$. Both cross-sectional $Z$-value analyses for the NIED data set (bottom row figures in fig. 3) display high $Z$-values (red) $5-20 \mathrm{~km}$ above the hypocenter, well within the rupture area outlined by the aftershock locations. By investigating the change in $Z$-values and cumulative number as a function of time, we estimated that this quiescence appeared 1.5 \pm 0.5 years before the mainshock. The highest $Z$-value of $Z=6.1$ is found in the NS trending cross-section, the highest $Z$-value in the EW cross-section is $Z=5.2$. Outside the rupture zone, $Z$-values were lower. The hypocenter itself displayed a $50 \%$ increase in the seismicity rate, leading to a negative $Z$-value of $Z=-3.1$. In fig. 5a-c we show LTA $(t)$ and the cumul-

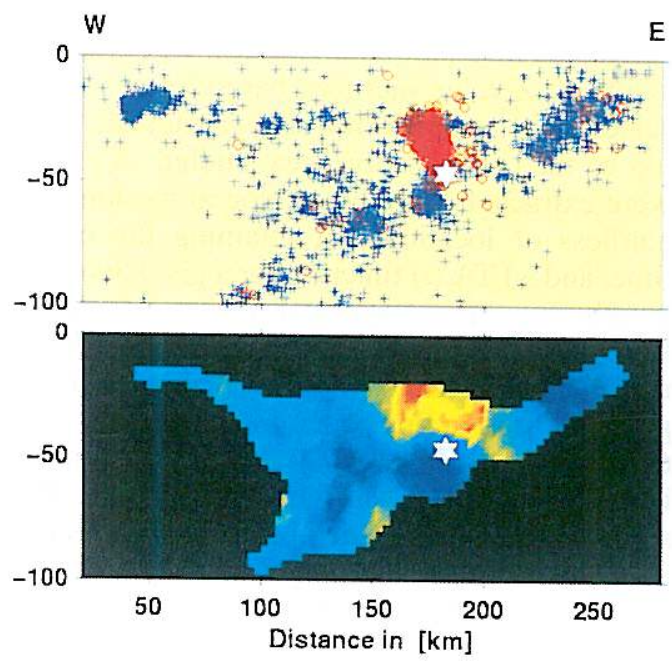

E $N$

S

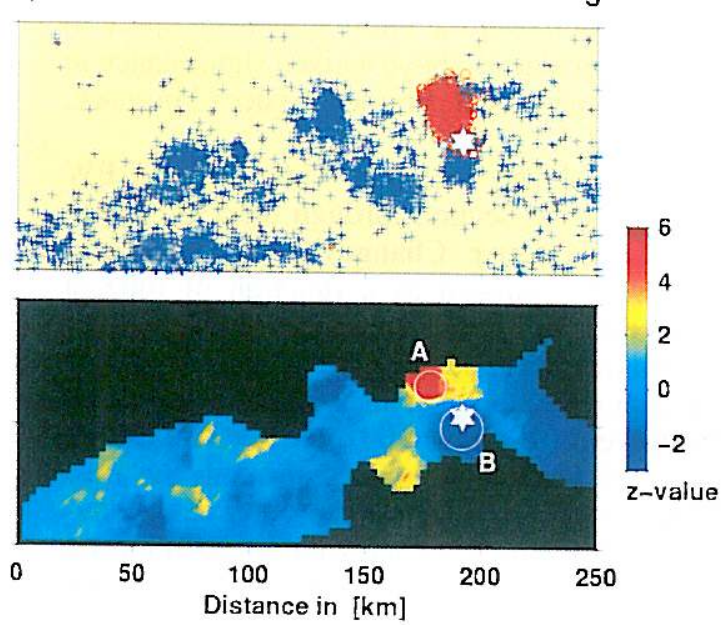

Fig. 3. Top left: EW trending cross-section through the hypocenter of the 1987 Chiba-toho-oki earthquake. marked by a star. Hypocenters are taken from the NIED data $(M \geq 2.2)$. The aftershocks of the 1987 mainshock within 30 days are plotted in red. Bottom left: spatial distribution of the standard deviate $Z$, computed for the EW trending cross-section shown in the top left. The $Z$-value compares the seismicity rate in the period 1986.461987.96 with the rate in the background period (1981.0-1997.0, but excluding the period 1986.46-1992.0). Positive $Z$-values indicate a seismicity rate decrease in the 1.5 years before the 1987 mainshock compared to the background rate and are shown in red. Sampled are the $N=100$ nearest earthquakes to each grid-node. Top right: NS trending cross-section through the 1987 hypocenter. Bottom right: Spatial distribution of the standard deviate Z, computed for the NS trending cross-section shown in the top right. 

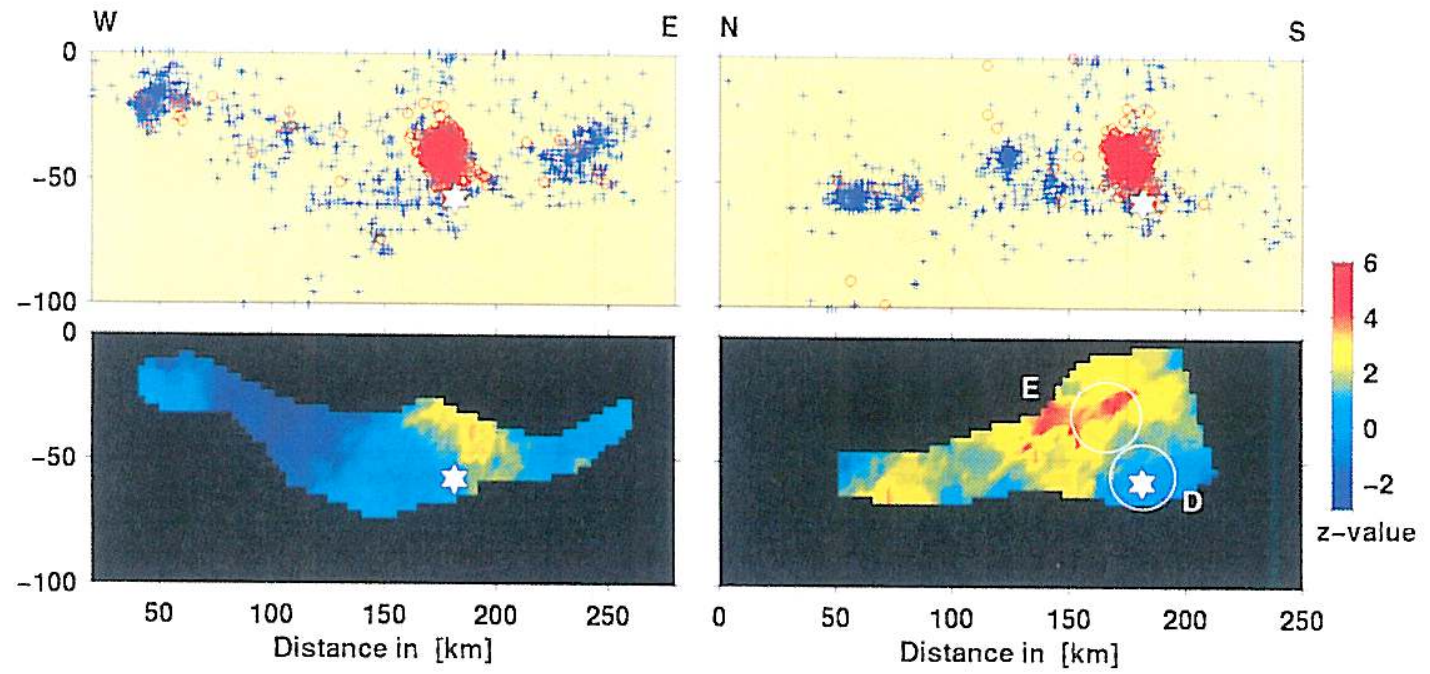

Fig. 4. The same as fig. 3, but using the JMA data set $(M \geq 2.5)$. Top left: EW trending cross-section through the hypocenter of the 1987 Chiba-toho-oki earthquake. Botlom left: Spatial distribution of the standard deviate Z, computed for the EW trending cross-section shown in the top left. The $Z$-value compares the seismicity rates in the period 1986.46-1987.96 with the rate in the background period (1981.0-1997.0, but excluding the period 1986.46-1992.0). Top right: NS trending cross-section through the 1987 hypocenter. Bottom right: spatial distribution of the standard deviate Z, computed for the NS trending cross-section shown in the top right.

ative number as a function of time for the area of strongest rate decrease and the hypocenter.

The JMA data displayed a somewhat similar distribution of $Z$-values (fig. 4). We used the same window length, $T_{w}=1.5$ years and $N=100$ used for the NIED data. The total number of events is 1120 and 1366 events in the NS and EW cross-section, which is reduced by over a factor two compared to the NIED data due to the higher magnitude of completeness of this cata$\log$. Consequently, the average radius of the sample volumes $(16 \mathrm{~km})$ is larger than those in the NIED data set. The strongest rate decrease of $Z=4.3$ can be identified in the NS crosssection above the 1987 mainshock hypocenter. The increase in the seismicity rate at the hypocenter location in the 1.5 years prior to the 1987 mainshock cannot be detected clearly in the JMA data, because of the generally small number of events around the hypocenter. Cumulative number graphs at selected locations are shown in fig. $5 \mathrm{~d}-\mathrm{f}$.

\subsection{Estimating the likelihood of random occurrence}

We then evaluated the likelihood of a quiescence of the maximum significance determined above $(Z=6.1)$ and with the optimized characteristics $\left(T_{1}=1.5, N=100\right)$ to occur at random. The analysis technique is outlined in the method section. We drew at random 1200 samples of 100 earthquakes each from the seismicity within the NS cross-section of the NIED and JMA data. For each time series, we computed the LTA $(t)$ time series for a window-length $T_{w}=1.5$ years and retained only the maximum $Z$-value. We repeated this process 1000 times, and plotted the histogram of $Z_{\text {mix }}$ values for both the JMA and NIED data set in fig. 6a,b. The 50percentile of this distribution represents the maximum $Z$-value we should on average expect for a particular, three-dimensional (distance, depth, time) $Z$-value matrix. For the NIED data set, we found a 50-percentile value of $Z_{\max }=6.76$, and $Z_{\max }(95 \%)=8.59$ (fig. 6a). The 

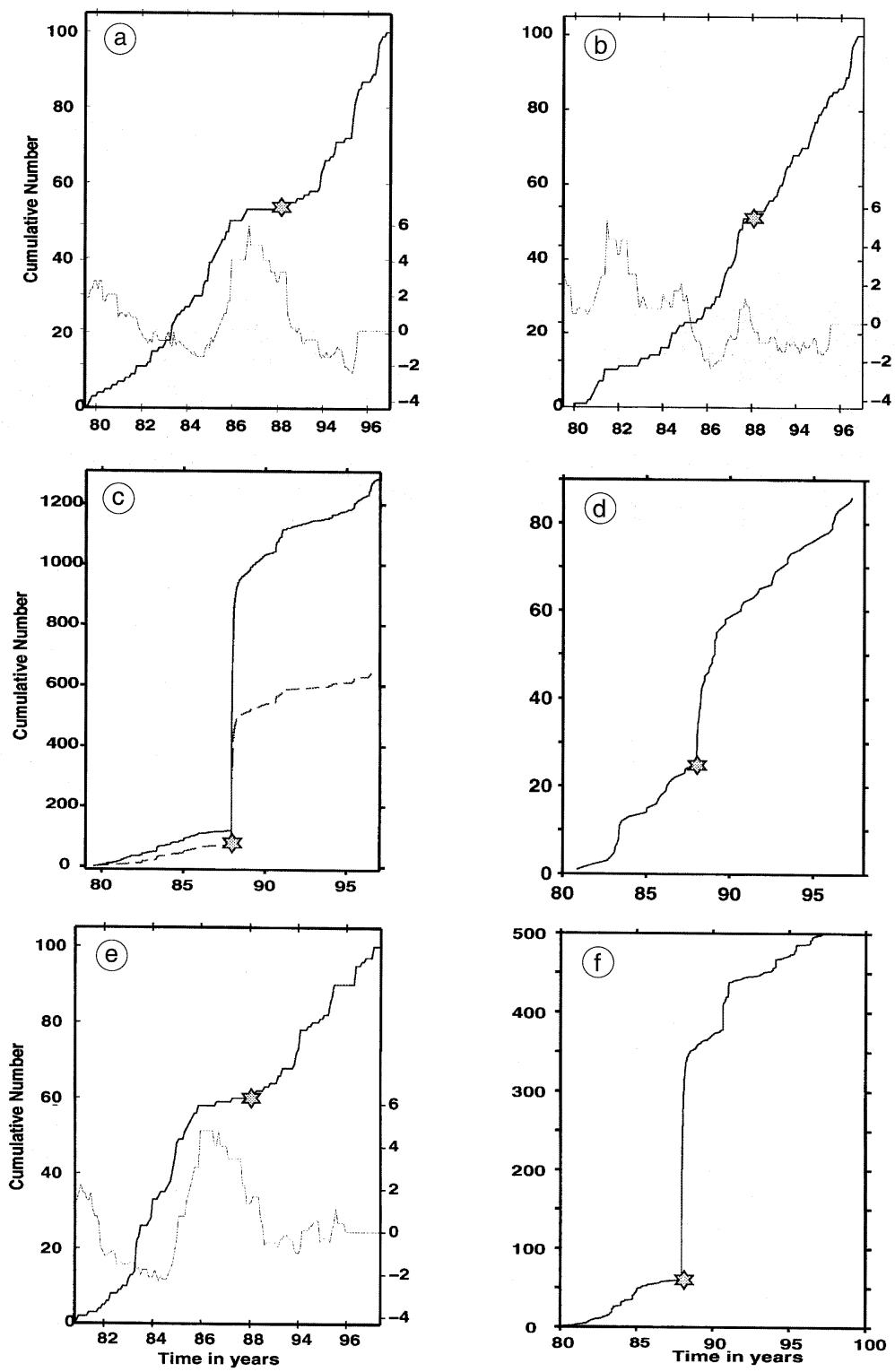

Fig. 5a-f. Cumulative number of earthquakes as a function of time for selected volumes. The thin line is the LTA $(t)$ time-series computed for a window-length $T_{w}=1.5$ years using earthquakes with $M \geq 2.2$ for NIED and $M \geq 2.5$ for JMA, unless specified. a) Shallow part of the 1987-rupture area, declustered NIED data (see fig. 3 for location). The years 1988.0-1992.0 are excluded from the graph. b) Hypocenter area of the 1987 mainshock, declustered NIED data. The years 1988-1992 are excluded from the graph. c) Shallow part of the 1987-rupture area, undeclustered NIED data. The solid line shows all magnitudes, the dashed line $M \geq 2.0$ only. d) Hypocenter area, declustered JMA data. e) Shallow part of the 1987-rupture area, declustered JMA data (see fig. 4 for location). The years 1988-1992 are excluded from the graph. f) Shallow part of the 1987-rupture area, undeclustered JMA data. 

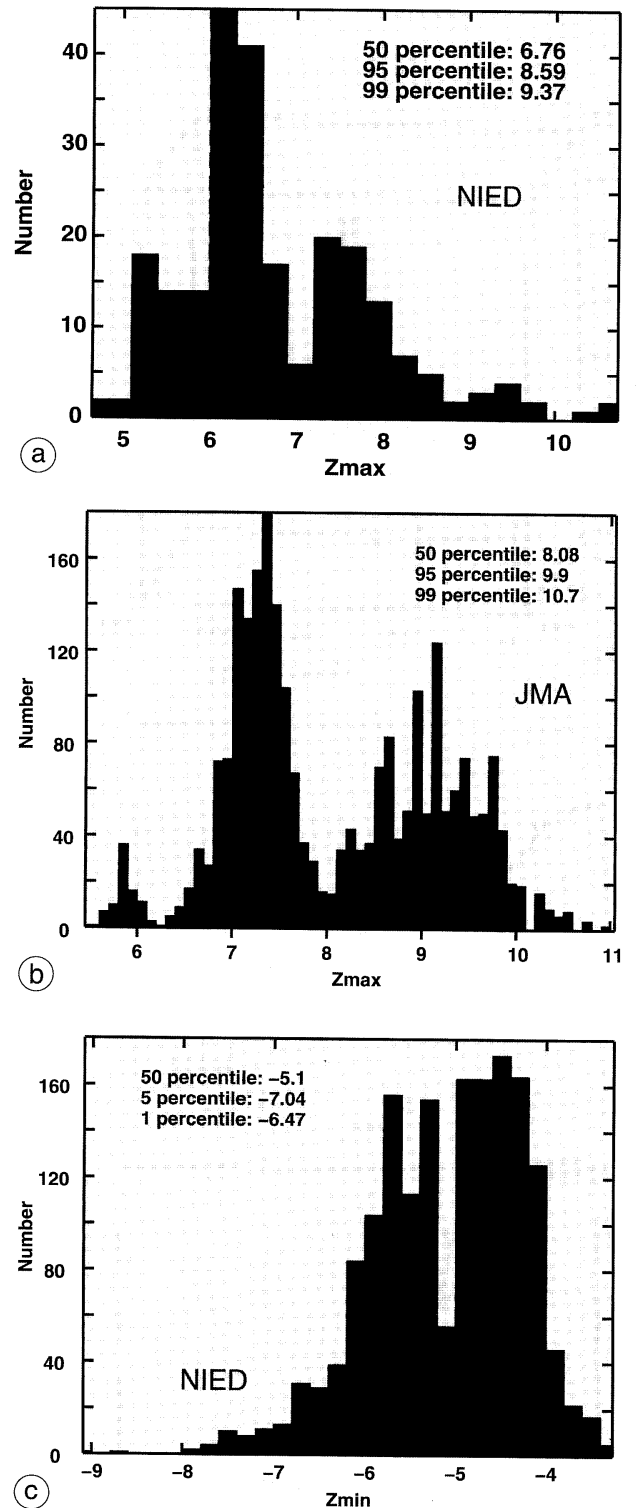

Fig. 6a-c. Histograms showing the distribution of the maximum and minimum $Z$-values. a) $Z_{\max }$ distribution computed from the NIED data using events within the NS trending cross-section. The 50, 95, and 99 percentile are given in the figure. b) $Z_{\max }$ distribution computed from the JMA data using events within the NS trending cross-section. c) $Z_{\min }$ distribution computed from the NIED data. The 50, 5, and 1 percentile are given in the figure. histogram does not represent a simple normal distribution but shows several distinct peaks $\left(Z_{\max }=6.2,7.5,9.4\right)$. This is a consequence of the discrete number of events contained within a quiet period of 1.5 years duration. A quiescence period can contain $0,1,2, \ldots$ events, and the $Z$-value varies around the peaks according to the standard deviation of the remainder of the time series. For the JMA data we found $Z_{\max }(50 \%)=8.08$ and $Z_{\max }(95 \%)=9.9$ (fig. 6b), somewhat higher than in the NIED data. For both catalogs we found that all of these values are considerably higher than the observed $Z$ values for the precursory quiescence (NIED: $Z_{\max }=6.1$, JMA: $Z_{\max }=4.3$ ).

In order to evaluate the significance of the seismicity rate increase around the hypocenter of the 1987 mainshock, we also performed a random minimum $Z$-value analysis. This analysis was analogous to the analysis for the decreased seismicity rate, but instead of retaining the maximum $Z$-value for each cumulative number plot, we retained the minimum $Z$-value (fig. 6c). We only performed this analysis for the NIED data set. The mean value of this distribution was $Z_{\min }(50 \%)=-5.1$ and $Z_{\min }(5 \%)=$ $=-7.04$ (fig. $6 \mathrm{c}$ ). These values are significantly lower than the observed $Z$-values for the rate increase around the hypocenter $(Z=-3.1)$.

\subsection{Alarm cube analysis}

The alarm cube analysis identifies all $Z$-values within the three-dimensional grid (distance, depth, time) of $Z$-values that exceed a given significance threshold $Z_{\text {alarm }}$ for a particular crosssection. Thus, we can find all occurrences in time and space of a quiescence at least as significant as the precursory quiescence under investigation. These we call false alarms. We only performed this analysis for quiescences, because, as shown in the above analysis, the rate increase detected before the mainshock is not significant and would produce a lot of false alarms. We used the same optimized values $(N=100$ and $T_{w}=1.5$ years) derived from the aforementioned analysis. The top row in fig. 7 shows the alarm cube for the NS cross-section at an alarm threshold of $Z_{\text {alarm }}=6.1$ for the NIED data and $Z_{\text {alarm }}=4.3$ 


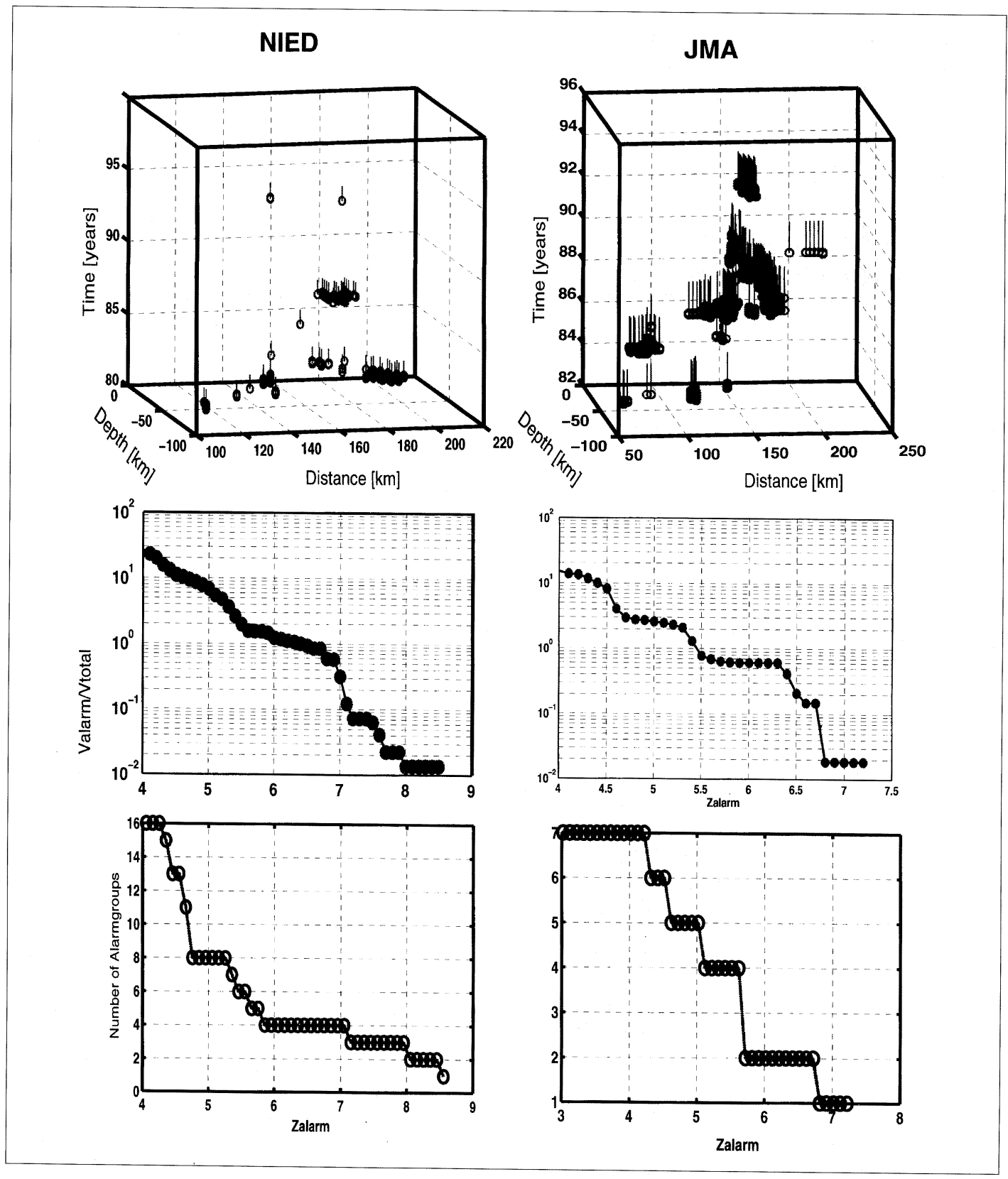

Fig. 7. Top: alarm cube for the NIED data (left) and the JMA data (right). A circle marks instance in space (distance versus depth) and time when the $z$-value exceeds the threshold value of $Z_{\text {alarm }}=6.1$ (NIED) and $Z_{\text {alarn }}=4.3$ (JMA). The $Z$-values are computed for a window length of $T_{w}=1.5$ years and a sampling radius corresponding to $N=100$ earthquakes. Middle: the ratio $V_{\text {alarm }} / V_{\text {total }}$ plotted as a function of alarm threshold $Z_{\text {alarm }}$ for the NIED data (left) and JMA data (right). Bottom: number of alarm groups as a function of $Z_{\text {alarm }}$. 
for the JMA data. Several alarms besides the precursory one can be identified, particularly in the JMA data. The ratio of the space-time volume covered by alarms to the total space-time volume for the investigated cross-section area and the number of alarm groups as a function of $Z_{\text {alarm }}$ are plotted in the middle and bottom row of fig. 7 , respectively. We concluded from these figures that in the NIED data (left column fig. 7) $75 \%$ (3 of 4 ) of the alarm-groups with a significance equal to or larger than the observed value of $Z=6.1$ are false alarms and the combined alarm volume occupies $1 \%$ of the total investigated volume. For the JMA data (right column in fig. 7), 83\% (5 of 6) of alarm-groups ranking equally or higher in significance than the precursory alarm are false alarms, and the combined alarm volume occupies more than $10 \%$ of the total investigated space-time volume.

\section{Discussion}

The seismicity rate in the $1.5 \pm 0.5$ years before the 1987 Chiba-toho-oki earthquake, Central Japan, is reduced compared to the background rate within the shallow part of the rupture zone of the mainshock. This decrease can be confirmed in two independently compiled earthquake catalogs (NIED and JMA, figs. 3 and 4). Our quantitative analysis is in agreement with the earlier study by Odaka and Maeda (1994) who described a precursory quiescence of 1-year duration based on a qualitative analysis of the JMA and University network data. The quiescence shows up more clearly in the higher quality and larger quantity NIED data set (e.g., fig. 5a,c). However, the quantitative analysis of the seismicity in the Kanto region reveals that the quiescence is neither unique nor highly unlikely to be created by chance (figs. $6 a, b$ and 7). The quiescence is surpassed in significance by several other quiescences within the same cross-section that are not precursors to subsequent mainshocks. The spatial and temporal correlation of the quiescence and the mainshock rupture area, as well as the fact that it can be observed in two independent catalogs suggests that we may be observing a precursory phenomenon. However, the quantitative signif- icance analysis (figs. 6a,b and 7) led us to reject the hypothesis that the decrease in the seismicity rate is a significant precursory seismic quiescence.

A three-step procedure was applied in this study to evaluate the significance of precursory seismic quiescence. For the 1987 Chiba-tohooki earthquake, we conclude that this event was not preceded by a significant precursory seismic quiescence. The significance level of three quiescences that are not precursors to subsequent mainshocks in the NIED catalog surpass that of the quiescence followed by the mainshock (fig. 7) even when we search only a 3 parameter space (distance, depth, $t$ ) and fix the optimized parameters $\left(T_{w}, N\right.$, location (NS crosssection)). A search over the entire six-dimensional parameter space $\left(X, Y, Z, T_{w}, N, t\right)$ would identify numerous additional periods of reduced seismicity of equal or higher significance. Thus, we conclude that one would be unable to detect the quiescence in real time monitoring. This is mainly due to the fact the background seismicity rate in the source area is too low to detect a quiescence of 1.5 years with a high enough confidence level. The entire rupture area as outlined by the aftershock zone (fig. 3) contains only a total of about 76 earthquakes in the declustered catalog in the 7 years before the mainshock. Although the entire shallow portion of the rupture area experienced a seismicity rate decrease of about $90 \%$ for a period of 1.5 years before the mainshock, the number of missing events is only about 10. This small number results in a low significance of the decrease, and such decreases are not uncommon in the data. In other studies the number of missing events was often much higher. For both the 1975 M 7.2 Kalapana event (Wyss et al., 1981) and the 1986 $M_{w} 8.0$ Adak event (Kisslinger, 1988), the seismicity rates in the precursory period were decreased by several hundreds of events when compared to the background rates. In these cases, the duration of the quiescence was 3-4 years, longer than in this study. On the opposite side of the spectrum, we find that for some mainshocks such as the $1995 M 7.2$ Kobe mainshock and the $1994 M 6.7$ Northridge event, the quiescence hypothesis as stated earlier cannot be tested at all because only a handful (Kobe: 30, North- 
ridge: 100) of micro-earthquakes are located within the immediate rupture area in the 10-15 years before the mainshocks. We consider it important to firmly establish the limitations of the applicability of the quiescence hypothesis.

The alarm cube analysis of the NIED data (fig. 7) indicates that $1 \%$ of the total space time volume is covered by alarms. One may think that the value of $1 \%$ is small enough for the anomaly to be considered significant. However, the process of optimizing parameters in order to identify an anomaly before the mainshock could easily produce such a low value by chance. If we apply Imoto's (Imoto, 1994) method of evaluating precursor performance to our case (1 target earthquake, 6 free parameters: location of cross-section, distance, depth, time, $N$, and $T_{w}$ ), we find that the space-time volume covered by alarms should be less than about $0.005 \%$ of the total space-time volume for an anomaly to be a more effective predictor than a random prediction. Therefore, the alarm cube analysis (fig. 7) confirms the $Z_{\max }$ analysis (fig. 6a,b) in suggesting that the precursory quiescence is not significant enough to be identifiable in real time as a precursory phenomena.

At the hypocenter location, we observed an increase in the seismicity rate in the 1.5 years before the 1987 Chiba-toho-oki earthquake in the NIED data (figs. 3 and 5b). The onset of the increased rate is approximately simultaneous to the onset of quiescence in the shallow part of the rupture zone. Foreshock activity in a broader sense, or an accelerated seismicity rate, has been proposed in a number of studies as a possible precursor (e.g., Shibazaki and Matsu'ura, 1995; Dodge et al., 1995; Abercrombie and Mori, 1996; Ogata et al., 1996; Bowman, 1997). If indeed precursory creep occurs on part of the fault plane (Dieterich, 1994), it could cause both an increased seismicity rate in one area and a decreased rate or quiescence in another. We therefore studied the increased seismicity rate in some detail, using techniques analogous to the ones established for the investigation of seismicity rate decreases. First we asked the question: how likely is an increased rate of the observed significance $(Z=-3.1)$ to occur by chance? We computed the distribution of the minimum $Z$-value $\left(Z_{\min }\right)$ (fig. 6c), using the declustered NIED cat- alog for the NS cross-section. The 50-percentile of this distribution is $Z_{\min }(50 \%)=-5.1$, indicating that rate increases as significant as the one under investigation cannot be distinguished from the phenomena caused by chance.

Next, we also used the alarm cube approach to identify periods of increased seismicity as or more significant than the increase observed at the hypocenter location. The minimum $Z$-value observed for the increased seismicity rate around the hypocenter reached $Z=-3.1$. The alarm cube at this significance level or higher showed numerous false alarms. Some of these false alarms are caused by aftershock sequences that are not entirely removed by the declustering process. However, the fact remains that increases in the seismicity rate as significant as the one before the 1987 mainshock are not uncommon in the data.

The fact that the decrease is observed in two independent catalogs, the spatial correlation of the increased rate with the subsequent mainshock hypocenter, and the simultaneous onset of quiescence and rate increase suggest a causal relationship with the mainshock. However, the significance analysis revealed that these phenomena can be created by chance and do occur in the actual data frequently without a subsequent mainshock. A further advancement of this analysis would require a simultaneous search for an increased and decreased seismicity pattern, which should be less likely to occur by chance.

\subsection{Artifacts in the JMA catalog}

The JMA catalog is widely used for analyzing seismicity in Japan, and earthquakes with magnitude $M \geq 3.0$ in the investigated region after 1979 were completely detected (fig. 2). However, we found that either an improvement of the observational system or a change of hypocenter determination algorithm caused a magnitude shift in 1989, which introduced an artificial change in the seismicity rate when analyzing only larger events. To investigate the homogeneity of reporting in more detail, we compared the $Z$-value maps derived from two catalogs, JMA and NIED (fig. 8). The Z-maps in the 

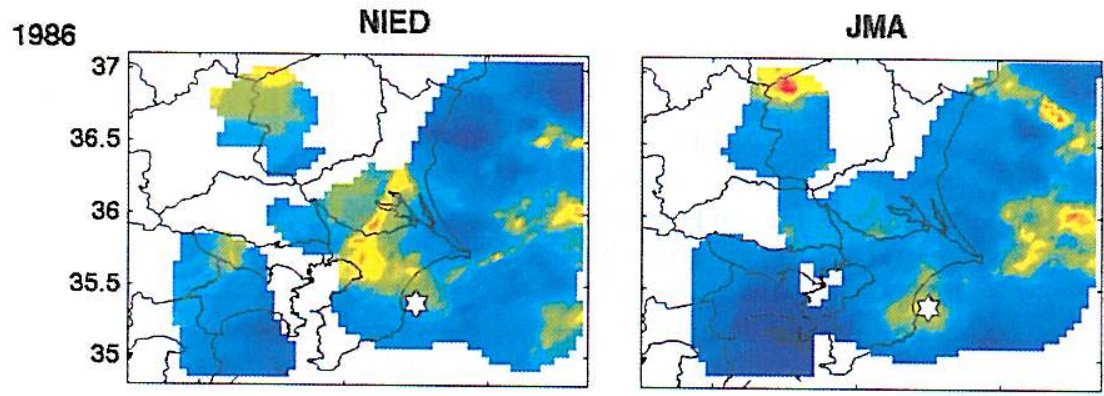

1989
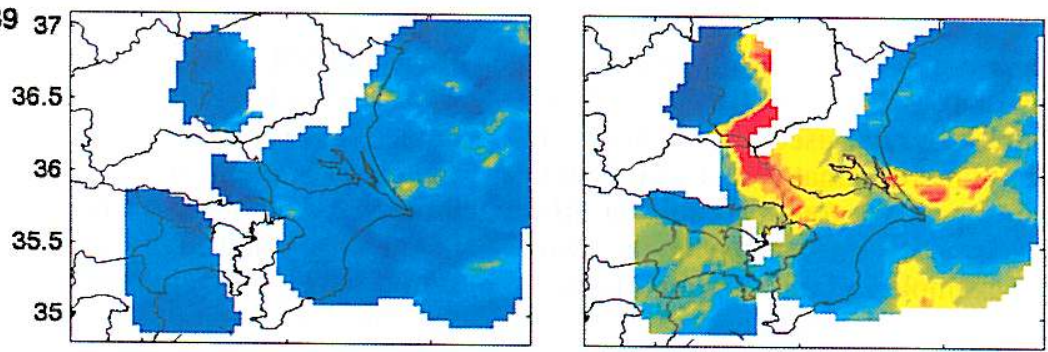

1992
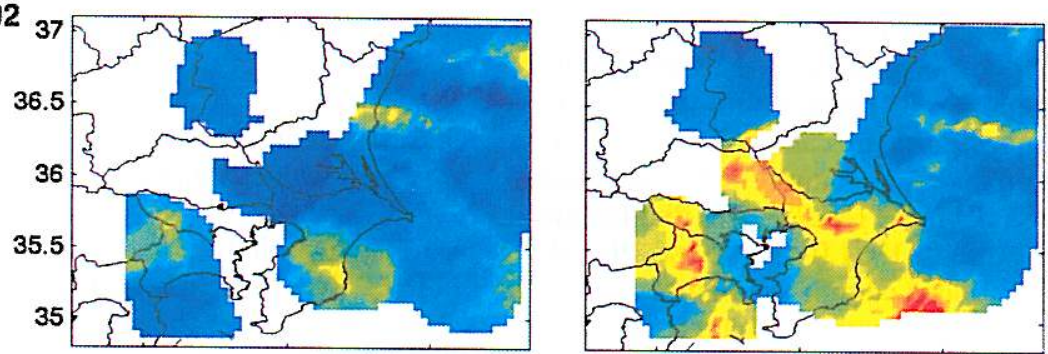

1995

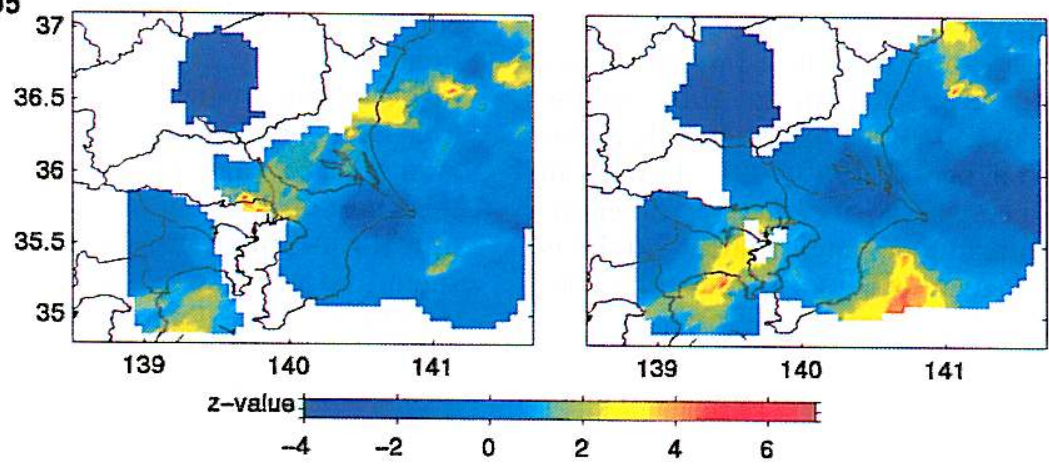

Fig. 8. Maps of the Kanto region showing the spatial distribution of the standard deviate $Z$ at four different times (1986, 1989, 1992 and 1995) for the NIED data with $M \geq 2.9$ and depth $\leq 45 \mathrm{~km}$ (left column) and JMA data with $M \geq 3.0$ and depth $\leq 50 \mathrm{~km}$ (right column). Z-values are computed by comparing the seismicity rate for a 2-year period (e.g., 1986.0-1988.0) with the overall seismicity rate (1981.0-1997.0, excluding the 2-year period). The $N=100$ nearest earthquakes to each grid-node are sampled, and nodes are only colored when the sampling radii is less than $50 \mathrm{~km}$. 
right column of fig. 8 are computed using the JMA catalog with magnitude $M \geq 3.0$ and depth $\leq 50 \mathrm{~km}$. The left column of fig. 8 shows the corresponding $Z$-maps based on the NIED cata$\log$ with magnitude $M \geq 2.9$ and depth $\leq 45 \mathrm{~km}$. The differences in the magnitude and depth thresholds used for the two catalogs are a result of our investigation of the systematic differences in hypocenter determination and magnitudes between the two catalogs. $Z$-value maps are computed for identical grid-nodes by sampling the $N=100$ nearest earthquakes and computing the LTA $(t)$ function for a window length $T_{w}=2$ years at four times (1986, 1989, 1992, 1995). For this comparison, we used the declustered catalogs and did not eliminate the 4 years after the 1987 mainshock. We found significant differences in seismicity rates between the two catalogs. For example, a large red area representing prominent quiescence appears in the JMA catalog during the periods 1989 and 1992 in the area centered at about $35.7^{\circ} \mathrm{N}$ and $140.2^{\circ} \mathrm{E}$, whereas no rate decrease was detected in the NIED catalog at the corresponding location (fig. 8). To investigate the cause of these differences, we sampled the seismicity within a $20 \mathrm{~km}$ radius from $35.7^{\circ} \mathrm{N}$ and $140.2^{\circ} \mathrm{E}$. We divided the data into two periods, 1981.0-1989.0 and 1989.01996.0, and produced cumulative number versus magnitude and number versus magnitude plots for each period (fig. 9). The cumulative number (top graphs) and the number (bottom graphs) for each period were normalized by the length of the periods. We found in the JMA catalog (left column of fig. 9) that the magnitude distribution for the period 1989-1996 showed an unusual shape, that is, the number of earthquakes with magnitude ranging about 2.6-3.3 is reduced compared with the number of earthquakes in the same magnitude bins in the earlier period. The magnitude distributions in the NIED catalog (right column of fig. 9) are almost identical for both periods. This suggests that the JMA catalog experienced a magnitude shift together with an overall improvement of detectability. Therefore, the rate decrease that appeared in the JMA catalog in this region is probably an artifact caused by a change in magnitude reporting. Since we cut the JMA data set at $M=3.0$ to produce the $Z$-value maps shown in fig. 8 , we artificially reduced the number of earthquakes in the later period. A number of similar artificial rate changes can be found in the JMA data set, and, to a lesser extent, also in the NIED data. However, we found no evidence that the quiescence in the rupture zone of the 1987 mainshock was caused by an artificial rate change.

At the epicenter location of the 1987 Chibatoho-oki earthquake (marked by a star in fig. 8) we found for the two year period before the mainshock (1986-1988) only a slight decrease in the seismicity rate $(Z=2.5 \sim 3.0)$ in both the JMA and NIED catalog. The decrease did not show up as clearly in the map views compared to the cross-sections (figs. 3 and 4), because we sampled different volumes and included volumes that did not show a rate decrease. It is also noteworthy that the only region which showed a somewhat high $Z$-value in both catalogs was the Sagami Bay region, located at $35.1^{\circ} \mathrm{N}$ and $139.3^{\circ} \mathrm{E}$ in the bottom figures and the activity rate there was still low at present.

\subsection{Implications for future monitoring of seismicity rate changes}

The quantitative analysis of the seismicity before the 1987 Chiba-toho-oki earthquake shows that neither the rate increase nor the rate decrease before the mainshock is significant enough to be detected as a precursor in a real time monitoring approach. For future monitoring, it is important to address the question of how large a rate change is needed to be considered an anomalous rate change. The answer to this question is useful for identifying anomalies in real-time monitoring of seismic activity that should be investigated more closely. We propose two criteria as a measure of significance in real-time: First, an anomaly should be at least as significant as all previously observed ones of the same duration and spatial extent, excluding identified artificial rate changes. An alarm cube analysis performed for the particular windowlength $T_{w}$ and sampling volume $N$ of the detected anomaly (fig. 7) should therefore show the anomaly uniquely. The second criterion is derived from the random $Z$-value analysis. For an anomaly to be considered outstanding, it should 

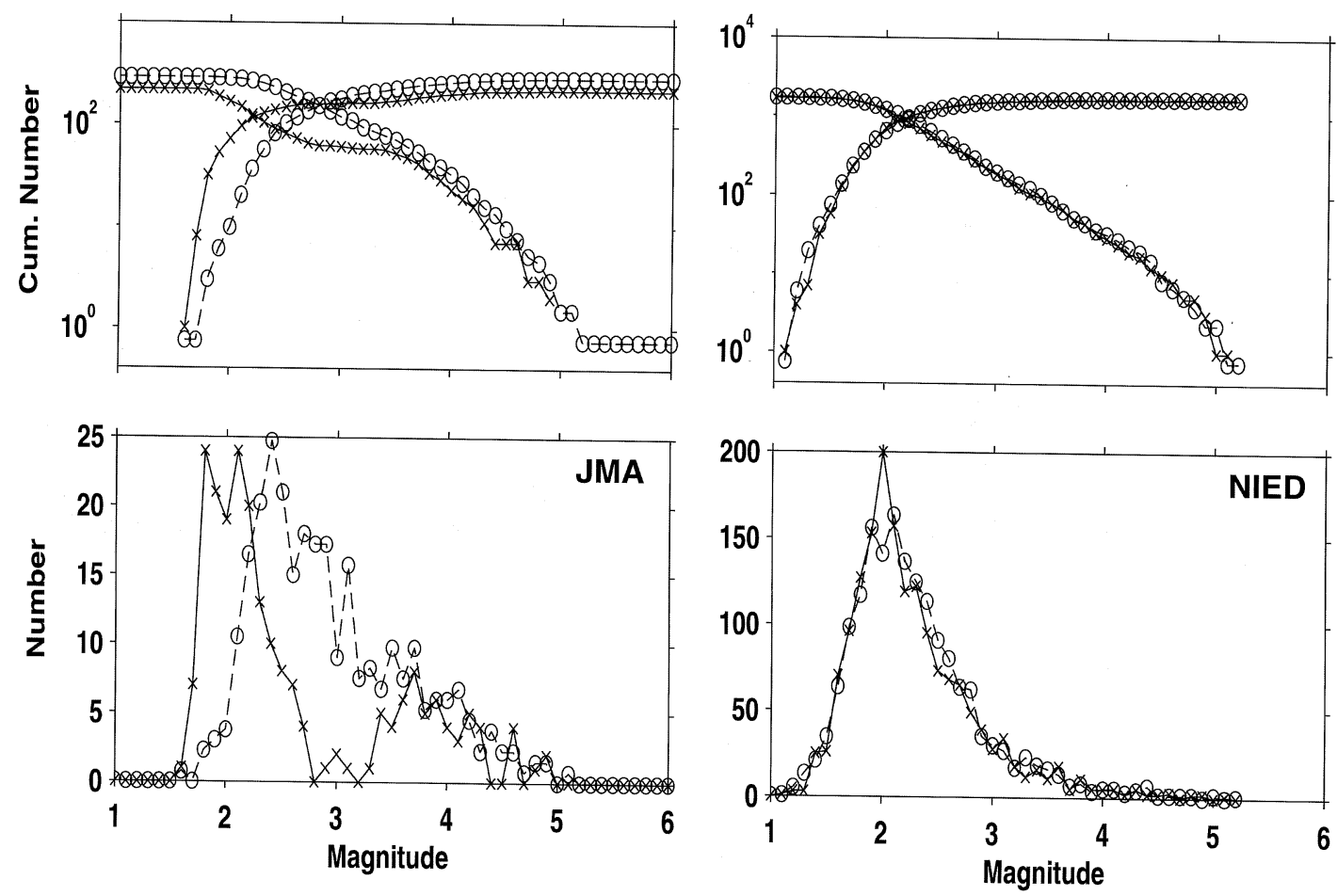

Fig. 9. Top: cumulative number as a function of magnitude plots using the JMA (left column) and NIED (right column) catalogs for the seismicity within a $20 \mathrm{~km}$ radius from $35.7^{\circ} \mathrm{N}$ and $140.2^{\circ} \mathrm{E}$. Circles and crosses denote the data for the periods of 1981.0-1989.0 and 1989.0-1996.0, respectively, each normalized by the length of the period. Bottom: number of events per magnitude bin for the same two periods.

have less than, for example, a 5\% probability of occurring by chance in the entire space-time volume under investigation. Since the $Z$-value, which reflects such a probability and is denoted by $Z(\mathrm{P} 95 \%)$ in this study, depends on both $T_{w}$ and $N$, we produced contour maps of the $Z^{w}$ (P95\%) level for the Kanto region for both the JMA and NIED data sets. The $Z$ (P95\%) values obtained for the cross-section data of the JMA and NIED catalogs are shown in fig. 10. To obtain the $Z$ (P95\%) values for the JMA catalog, we used only the data after 1990 to reduce the effects of artifacts, and used the earthquakes with magnitude $M_{c} \geq 2.5$ for that period. The dependency of $Z$ (P95\%) on $T_{w}$ and $N$ (fig. 10) shows some general features of interest: for constant $N$, the absolute $Z$-value decreases with increasing $T_{w}$. From fig. 10, we can determine the $Z$-value that indicates a significant anomalous rate change for the optimized parameter values of $N$ and $T_{w}$. For example, the $Z$-values of $95 \%$ significance of a quiescence and rate increase that are required for the NIED catalog in the case of $N=200$ and $T_{w}=2.5$ years are about $Z=6.5$ and -5.6 , respectively. We consider the $Z(\mathrm{P} 95 \%)$ value a useful parameter for the evaluation of future seismicity rate changes.

To further improve our ability to study the phenomena of both precursory seismic quiescence and increased seismicity rates, it would be helpful to improve the 'signal to noise' ratio in earthquake catalogs. As far as the quality and quantity of data used in the study region are not improved, we are currently not able to resolve a quiescence period less than 1.5 years even if it affects the entire rupture area of the $M 6.7$ main- 

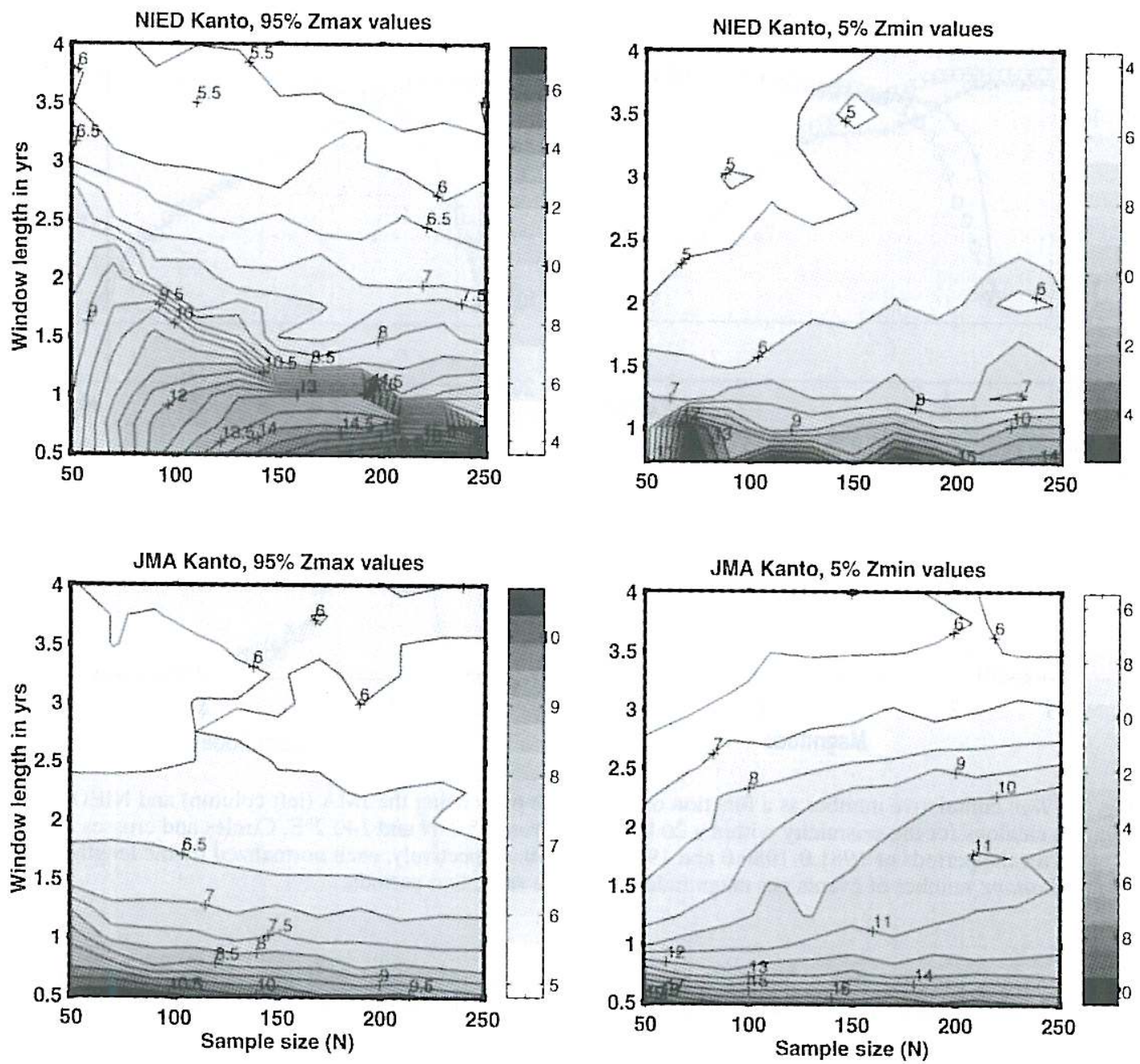

Fig. 10. Contour maps of the 95-percentile $Z_{\max }$ value (left columns) and the 5-percentile $Z_{\text {min }}$ value (right columns). The $Z_{\text {max }}$ and $Z_{\text {min }}$ values are plotted as a function of sample size $N$ and window length $T_{w}$. The top row uses the NIED data for the entire period and $M \geq 2.2$ within the NS cross-section, and the bottom row the JMA data for the period after 1990 and $M \geq 2.5$. An observed $Z$-value above the $95 \% Z_{\max }$ values indicates that the decrease has a less than $5 \%$ probability to be created by chance.

shock (20-30 km length). Improved monitoring (i.e. lower $M_{r}$ ) can strengthen the signal, that is, the number of missing or added events. Howev$\mathrm{er}$, it is also necessary to reduce the 'noise' introduced into earthquake catalogs. Many of the fluctuations in seismicity rates can be traced back to changes in the monitoring system, such as the introduction or removal of stations, modification of event analysis procedures, etc. Close attention should be paid to reduce these artificially introduced rate changes, or at least to identify them as such. 


\section{Conclusions}

Seismic quiescence appeared $1.5 \pm 0.5$ years before the mainshock in the shallow part of the focal region of the 1987 Chiba-toho-oki earthquake. This quiescence is observed in both the JMA $(M \geq 2.5)$ and NIED $(M \geq 2.2)$ catalogs. A quantitative analysis of the significance level of the quiescence, however, suggests that we cannot distinguish the precursory seismic quiescence from background fluctuation in seismicity rate at a high enough confidence level.

- The seismicity rate in the hypocentral area increased by over $50 \%$ in the 1.5 years before the mainshock as compared to the background (1981-1986.4). The onset of the quiescence and the rate increase are simultaneous and generally consistent with a model of precursory creep on the fault plane. However, the quantitative analysis of the likelihood and distribution of seismicity rates increases shows that the rate increase before the mainshock cannot be detected uniquely in the data set.

- The JMA catalog contains many artificially introduced rate changes and magnitude shifts.

- Contour maps of the $Z$ (P95\%) values as a function of window length $T_{w}$ and sampling size $N$ are introduced. These contour maps can aid us in identifying significant rate changes that should be analyzed more closely in real time monitoring.

\section{Acknowledgements}

The authors would like to thank Max Wyss, Hilary Fletcher and Clive S. Langham for their helpful comments and suggestions. We are grateful to the National Institute for Disaster Prevention for providing the earthquake catalog investigated. This work was partly supported by a Science and Technology Agency of Japan fellowship (SW).

\section{REFERENCE}

ABERCROMbIE, R.E. and J. MORI (1996): Occurrence patterns of foreshocks to large earthquakes in the western United States, Nature, 381, 303-307.

BOWMAN, J.R. (1997): A seismic precursor to a sequence of
$M_{s}$ 6.3-6.7 midplate earthquakes in Australia, Pageoph, 149, 61-78.

DIETRICH, J.H. (1994): A constitutive law for rate of earthquakes production and its application to earthquake clustering, J. Geophys. Res., 99, 2601-2618.

DieTERICH, J.H. and P.G. Okubo (1996): An unusual pattern of seismic quiescence at Kalapana, Hawaii, Geophys. Res. Lett., 23, 447-450.

DODGE, D.A., G.C. BEROZA and W.L. ELLSWORTH (1995): The foreshock sequence of the Landers, California earthquake and its implications for earthquake nucleation, J. Geophys. Res., 100, 9865-9880.

ENEVA, M., R.E. HABERMANN and M.W. HAMBURGER (1995): Artificial and natural changes in the rates of seismic activity: a case study of the Garm region, Tajikistan (CIS), Geophys. J. Int., 116, 157-172.

HabermanN, R.E. (1987): Man-made changes of Seismicity rates, Bull. Seismol. Soc. Am., 77, 141-159.

Imoto, M. (1994): Information Criterion of Precursors, J. Seismol. Soc. Jpn., 47, 137-142.

INOUYE, W. (1965): On the seismicity in the epicentral region and its neighborhood before the Niigata earthquake, $Q$. J. Seismol., 29, 31-36.

ISHIKAWA, Y. (1987): Change of JMA hypocenter data and some problems, Q. J. Seismol., 51, 47-56.

KISSLINGER, C. (1988): An experiment earthquake prediction and the 7 May 1986 Andreanof Islands earthquake, Bull. Seismol. Soc. Am., 78, 218-229.

MoGI, K. (1969): Some features of recent Seismic activity in and near Japan (2), Activity before and after great earthquakes, Bull. Earthquake Res. Inst., Univ. Tokyo, 47, 395-417.

MoGI, K. (1985): Precursors to the 1983 Japan Sea earthquake, Earthquake Prediction Research, 3, 493-517.

NoGUCHI, S. (1983): Spatio-temporal patterns of seismicity and some features of recent seismic activity off Ibaraki prefecture, eastern Japan, and adjacent regions, Zisin, 36, 619-634.

ODAKA, T. and K. MAEDA (1994): Changes in seismic activities in the focal region and nearby seismic nests before the 1987 Chiba-toho-oki earthquake, J. Seismol. Soc. Jpn., 47, 365-374.

OGATA, Y. (1992): Detection of precursory relative quiescence before great earthquakes through a statistical model, J. Geophys. Res., 97, 19845-19871.

OGATA, Y., T. UTSU and K. KATSURA (1996): Statistical discrimination of foreshocks from other earthquake clusters, Geophys. J. Int., 127, 17-30.

OKADA, Y. (1984): First results from Japanese network for earthquake prediction, Nature, 312, 500-501.

OKADA, Y. and K. KASAHARA (1990): Earthquake of 1987, off Chiba, Central Japan and possible triggering of Eastern Tokyo earthquake of 1988, Tectonophysics, 172, 351-364.

REASEnBERG, P.A. (1985): Second-order moment of Central California Seismicity, J. Geophys. Res., 90, 5479-5495.

Reasenberg, P.A. and M.V. Matthews (1988): Precursory seismic quiescence: a preliminary assessment of the hypothesis, Pageoph, 126, 373-406.

SHIBAZAKI, B. and M. MATSU'URA (1995): Foreshocks and pre-events associated with the nucleation of large 
earthquakes, Geophys. Res. Lett., 22, 1305-1308.

StEIN, R.S., G.C.P. KING and J. LIN (1992): Change in failure stress on the southern San Andreas fault system caused by the 1992 Magnitude $=7.4$ Landers earthquake, Science, 258, 1328-1332.

Toda, S., R. Stein, P. ReasenberG, J. Dietrich and A. Yoshida (1998): Stress transferred by the 1995 $M_{w}=6.9$ Kobe, Japan, shock: effect on aftershocks and future earthquake probabilities, J. Geophys. Res., 103, 24543-24565.

WIEMER, S. (1996). Analysis of seismicity: new techniques and case studies, Dissertation Thesis, University of Alaska, Fairbanks, Alaska, pp. 151.

WIEMER, S. and M. WySS (1994): Seismic quiescence before the Landers $(M=7.5)$ and Big Bear $(M=6.5)$ 1992 earthquakes, Bull. Seismol. Soc. Am., 84, 900-916.

WYSS, M. (1986): Seismic quiescence precursor to the 1983 $(M=6.6)$, Hawaii, earthquake, Bull. Seismol. Soc. Am., 76, 785-800.

WYSS, M. (1991): Reporting history of the central Aleutians Seismograph network and the quiescence preceding the 1986 Andreanof Island earthquake, Bull. Seismol. Soc. Am., 81, 1231-1254.

WYSS, M. and R.O. BURFORD (1987): A predicted earthquake on the San Andreas fault, California, Nature, 329, 323-325.

WySS, M. and R.E. HABERMANN (1988): Precursory seismic quiescence, Pure Appl. Geophys., 126, 319-332.
Wyss, M. and S. WIEMER (1997): Two current seismic quiescences within $40 \mathrm{~km}$ of Tokyo, Geophys. J. Int., 128, 459-473.

Wyss, M., F.W. KLEIN and A.C. JOHNSTON (1981): Precursors to the Kalapana $M=7.2$ earthquake, J. Geophys. Res., 86, 3881-3900.

Wyss, M., K. ShimazaKi and T. Urabe (1996): Quantitative mapping of a precursory quiescence to the Izu-Oshima 1990 (M6.5) earthquake, Japan, Geophys. J. Int., 127, 735-743.

Wyss, M., R. CONSOLE and M. MurRu (1997): Seismicity rate change before the Irpinia $(M=6.9) 1980$ earthquake, Bull. Seismol. Soc. Am., 87, 318-326.

Wyss, M., A. Hasegawa, S. Wiemer and N. Umino (1999): Quantitative mapping of precursory seismic quiescence before the $1989, M 7.1$ off-Sanriku earthquake, Japan, Ann. Geofis., 42 (5), 851-869.

YAMASHINA, K. and Y. INOUE (1979): A doughnut-shaped pattern of seismic activity preceding the Shimane earthquake of 1978, Japan, Nature, 278, 48-50.

YoshidA, A., H. ITO and K. Hosono (1996): Precursory seismic quiescence appearing along tectonic zone just before the occurrence of intraplate earthquake, $J$. Geogr., 105, 15-25.

ZUNIGA, R. and M. WYSS (1995): Inadvertent changes in magnitude reported in earthquake catalogs: influence on $b$-value estimates, Bull. Seismol. Soc. Am., 85, 18581866. 